\title{
UNE COCCIDIE DE SERPENT
}

\section{Par W. L. YAKIMOFF et F. F. GOUSSEFF}

Nous avons déjà décrit, en 1934, la coccidie d'un serpent (Elaps quatuor-lineata sauromates), dont les oocystes avaient une forme octogonale et nous lui avons donné le nom d'Isospora phisalix.

Nous avons trouvé chez le même serpent une autre coccidie, chez laquelle la forme des oocystes est ronde ou ovale. Elle est incolore, possède une membrane à double contour et n'a point de micropyle. Le tableau ci-dessous indique les dimensions des 58 oocystes ronds qui ont été mesurés.

\section{Formes RONDES}

$\begin{array}{llllll}\text { Nombre d'oocystes examinés : } & 13 & 11 & 18 & 16 & \text { Total : } 58\end{array}$ Dimensions en $\mu \ldots \ldots \ldots \ldots$ : $\quad \begin{array}{lllll}18 & 20 & 22 & 24 & \text { Moyenne : } 20,2\end{array}$

D'après ce tableau, on voit que les dimensions des oocystes varient de 18 à $24 \mu$, la moyenne étant de $20 \mu, 2$. Le protoplasme est réduit à une sphère de $16 \mu$ de diamètre.

\section{Formes ovales}

\begin{tabular}{|c|c|c|c|c|c|c|c|c|}
\hline $\begin{array}{l}\text { Nombre } \\
\text { d'oocystes } \\
\text { examinés }\end{array}$ & \} & 13 & 28 & 26 & 15 & 14 & 12 & 11 \\
\hline $\begin{array}{c}\text { Dimensions } \\
\text { en } \mu\end{array}$ & $16 \times 13$ & $20 \times 16$ & $20 \times 17$ & $22 \times 20$ & $24 \times 20$ & $25 \times 22$ & $26 \times 20$ & $26 \times 22$ \\
\hline $\begin{array}{l}\text { Rapport du } \\
\text { grand axe } \\
\text { au petit axe }\end{array}$ & 0,81 & 0,84 & 0,85 & 0,90 & 0,83 & 0,88 & 0,76 & 0,84 \\
\hline
\end{tabular}

Nous voyons done que les dimensions de la forme ovale sont : 16-26 $\mu \times 13-22 \mu$, ce qui donne une moyenne de $22 \mu, 1 \times 18 \mu, 7$; les dimensions le plus souvent rencontrées sont : $20 \mu \times 17 \mu$, les dimensions maxima étant de $26 \mu \times 22 \mu$ et les dimensions minima de $16 \mu \times 13 \mu$.

Annales de Parasitologie, T. XIII, $\mathrm{N}^{\circ}$ 1. - Janvier 1935, p. 28-31. 
Le rapport du grand axe au petit axe est de : $1: 0,78-0,90$ et en moyenne de $1: 0,84$, le rapport le plus fréquent étant de $1: 0.85$.

Nous pensons que ces deux formes, rondes et ovales, appartiennent à la même coccidie.

Nous avons mis les excréments dans une solution de bichromate de potasse à 2,5 p. cent. Dans les deux formes, il se développe 2 spores et un granule polaire. Les dimensions de ces spores sont de

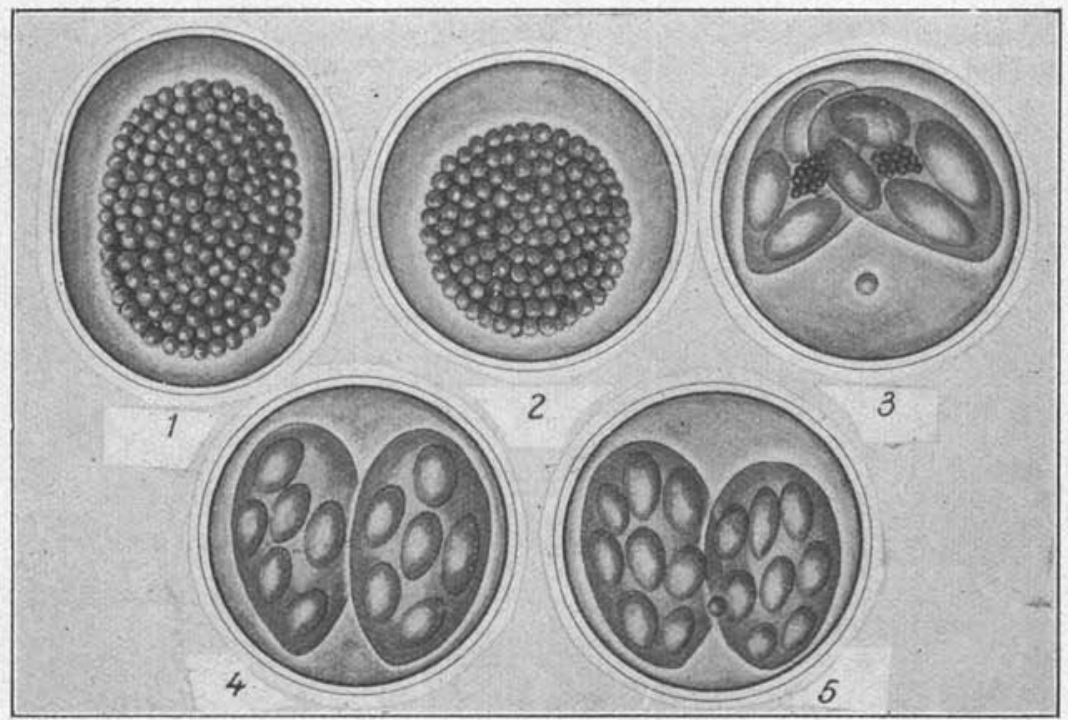

FIG. - Dorisiella hoarei n. sp. 1, forme ovale ; 2 , forme ronde; 3 , forme ronde avec deux spores et quatre sporozoites; 4 , forme ronde avec deux spores et six sporozoïtes ; 5 , forme ronde avec deux spores et huit sporozoïtes.

14 à $16 \mu \times 8 \mu$. Puis, dans ces spores, apparaissent des sporozoïtes au nombre de 4, 6 ou 8 . L'oocyste n'a pas de reliquat; les sporocystes ayant 4 sporozoïtes en ont un, mais nous n'en avons pas vu dans les sporocystes à 6 et 8 sporozoïtes.

Nous avons done devant nous un disporocystidé, hécadécazoïque. Ce diagnostic est basé sur les formes ayant un nombre maximum de sporozoïtes (8) dans un sporocyste. Etant donné que chaque spore peut contenir soit 4 , soit 6 ou 8 sporozoïtes, cette coccidie peut présenter des formes du type Dorisiella ( 8 sporozoïtes), aussi bien que des formes du type Isospora (4 sporozoïtes), et, de plus, des formes 
intermédiaires à 6 sporozoïtes. Pour pouvoir discerner laquelle de ces trois formes est la forme typique du parasite, les autres étant des exceptions, il faudrait déterminer dans quel pourcentage on rencontre chacune d'elles. Nous n'avons malheureusement pas la possibilité d'effectuer ces recherches en ce moment. D'après les données que nous possédons, nous pouvons aussi bien dire que nous sommes en présence d'une coccodie du genre Isospora avec 6 et 8 sporozoïtes aberrants que d'une coccidie du genre Dorisiella ayant exceptionnellement 4 et 6 sporozoïtes.

Le genre Dorisiella a été créé par Ray en 1930. Les représentants de ce genre furent d'abord trouvés dans les cellules épithéliales de l'intestin de Scolelepis fuliginosa, d'où le nom de l'espèce-type : D. scolelepidis. Ce parasite a deux spores dont chacune contient 8 sporozoïtes.

Cependant, entre Dorisiella scolelepidis et notre coccidie, il existe une certaine différence. Ray avait vu son parasite dans les cellules épithéliales de l'intestin, mais il n'a pas constaté que les deux spores fussent enveloppées par une membrane commune.

La question de la présence ou de l'absence de cette membrane chez les oocystes de Dorisiella et d'autres coccidies, en particulier chez les oocystes d'Isospora, a été étudiée à fond par Hoare (1933). Sans doute, l'épaisseur de la paroi de l'oocyste varie considérablement, même chez les individus d'une même espèce, par exemple chez l'Isospora, et peut, dans certains cas, disparaitre complètement, comme chez la Dorisiella scolelepidis, mais ceci ne doit pas avoir d'influence sur la classification de l'espèce.

Nous avons trouvé notre parasite dans les excréments et, ainsi qu'on le voit sur les figures, il a, comme beaucoup d'autres oocystes de coccidie, une enveloppe à double contour et deux spores. On sait qu'il existe des oocystes, comme dans l'Isispora dirumpens Hoare 1933, dont l'enveloppe très fine est évidemment à un seul contour.

Le genre Dorisiella Ray 1930 occupe, dans la classification des coccidies de Hoare (1933), une place située entre les genres Isospora et un hypothétique disporocystidé polyzoïque d'une part et, d'autre part, entre un problématique monosporocystidé hécadécazoïque et le genre Wenyonella.

Tant que les points ci-dessus mentionnés ne seront pas éclaircis, nous ne pouvons pas établir, d'une façon formelle, que notre coccidie appartient au genre Dorisiella, et c'est pour cette raison que nous ne la plaçons que provisoirement dans ce genre. Pour le cas où l'avenir confirmerait ce classement, nous nommons cette coccidie Dorisiella hoarei n. sp., en l'honneur du protozoologiste 
anglais C. A. Hoare, que nous remercions très vivement pour la consultation qu'il a bien voulu nous donner à propos de cette coccidie.

\section{Bibliographie}

Hoare (C. A.). - Studies on some new ophidian and avian coccidia from Uganda, with a revision of the classification of the Eimeriidx. Parasitology, XXV, 1933, p. 359-388.

Ray (H. N.), - Studies on some Sporozoa in polychæte worms. Parasitology, XXII, 1930 , p. 370.

Y gencoccidie. Arch. $f$. Protistenk., LXXXI, 1934, p. 547-550. 